\title{
Rupture of the Unscarred Gravid Uterus: Case Series
}

\author{
Robert O’Sullivan ${ }^{\mathrm{a}, \mathrm{b}}$, Craig Sauer ${ }^{\mathrm{a}}$, Perry Friedman ${ }^{\mathrm{a}}$, Mitchell Clark ${ }^{\mathrm{a}}$, \\ Gulden Menderes ${ }^{\mathrm{a}}$
}

\begin{abstract}
Rupture of the unscarred uterus is estimated to occur in approximately 0.8 in 10,000 deliveries. It is a potentially catastrophic event for both mother and fetus. We present two such cases and review the relevant literature. The first case concerns a multiparous patient who attended due to reduced fetal movements and subsequently incurred fetal demise, sepsis and significant hemorrhage. The second case concerns a patient in her second delivery who developed uterine rupture after a prolonged second stage of labor. We recommend that clinicians maintain a high index of suspicion for uterine rupture even in the absence of prior cesarean delivery.
\end{abstract}

Keywords: Unscarred; Uterus; Uterine rupture

\section{Introduction}

Rupture of an unscarred gravid uterus is a rarely encountered event in clinical practice [1]. It is a challenging diagnosis that has significant maternal and fetal morbidity and mortality. Herein, we present two cases of uterine rupture with distinct clinical courses and management.

\section{Case Reports}

\section{Case 1}

A 36-year-old G8P3043 from Pakistan presented at 38 weeks and 2 days gestation complaining of decreased fetal movement and contractions. This pregnancy was complicated by

Manuscript accepted for publication June 03, 2015

aDepartment of Obstetrics and Gynecology, Bridgeport Hospital, 267 Grant St, Bridgeport, CT 06610, USA

bCorresponding Author: Robert O'Sullivan, Department of Obstetrics and Gynecology, Bridgeport Hospital, 267 Grant Street, Bridgeport, CT 06610, USA. Email: robbie25@gmail.com

doi: http://dx.doi.org/10.14740/jmc2203w diet controlled gestational diabetes. The obstetric history included three uncomplicated vaginal deliveries at term and four first trimester miscarriages, three of which required uterine curettage, without any reported complications. Serial vaginal examinations demonstrated the absence of cervical dilatation and continuous fetal heart monitoring demonstrated fetal wellbeing. An advanced ultrasound demonstrated a biophysical profile score of $8 / 8$. The patient reported an improvement in symptoms and was discharged home in stable condition.

The following day, she attended her obstetrician and reported generalized malaise and back pain. The fetal heart rate was determined to be approximately 40 beats minute. The patient was transferred to the hospital, and ultrasound demonstrated the absence of fetal heart tones. Maternal vital signs were temperature of $98.4{ }^{\circ} \mathrm{F}$, pulse of 72 beats/min, respiratory rate of 18/min, and blood pressure of 118/56 $\mathrm{mm} \mathrm{Hg}$. Lab results revealed $\mathrm{Hb} / \mathrm{Htc}$ of $9.7 \mathrm{~g} / \mathrm{dL}$ and $28.1 \%$, PT of 10.4 , PTT of 22.2, fibrinogen of 415, INR of 0.96 , and lactate of $3.6 \mathrm{mg} /$ dL. Blood and urine cultures showed no growth.

The differential diagnosis included abruption, hypovolemia and sepsis. The patient received $3 \mathrm{~L}$ of lactated ringers fluid through a central line prior to delivery. Patient was examined and was $2 / 80 /-3$ with bulging membranes. The patient agreed to an induction of labor with oxytocin, and received an epidural for pain management. Upon artificial rupture of membranes, there was a thick, green, non-odorous fluid noted.

The patient continued to develop worsening tachycardia despite the administration of intravenous fluids. Laboratory findings showed hemoglobin of $7.4 \mathrm{~g} / \mathrm{dL}$, white cell count of 20.6 cells $/ \mu \mathrm{L}$, lactic acid of $5.8 \mathrm{mg} / \mathrm{dL}$, fibrinogen of $133 \mathrm{mg} /$ $\mathrm{dL}$ and platelets of $106 \times 10^{9} / \mathrm{L}$. She was started on piperacillin-tazobactam and gentamicin for presumptive sepsis, and the patient was transferred to the intensive care unit. The patient was unable to effectively push due to maternal exhaustion; therefore, a forceps-assisted vaginal delivery was successfully performed. The placenta was manually extracted and did not demonstrate any evidence of retroplacental clot. Total blood loss at delivery was estimated to be $300 \mathrm{~mL}$.

Subsequently, the patient's condition improved but serial complete blood counts identified a progressive decline in hemoglobin to $8.2 \mathrm{~g} / \mathrm{dL}$ and hematocrit to $23.7 \%$ despite the administration of seven units of packed red blood cells. The patient was noted to have significant abdominal distension and discomfort. A computerized tomography of the abdomen 
and pelvis was concerning for right-sided uterine rupture and showed a $13.5 \times 3 \mathrm{~cm}$ right-sided pelvic hematoma with large amount of hemoperitoneum. Given the patient's hemodynamic stability, diagnostic laparoscopy was performed. Four liters of blood were initially evacuated, and a $4-\mathrm{cm}$ right fundal uterine rupture was noted. The defect was repaired in two layers using barbed sutures.

The patient tolerated the procedure well and was discharged home on postoperative day 4 in stable condition. The placental pathology revealed no evidence of chorioamnionitis, abruption or other abnormality.

\section{Case 2}

A 35-year-old, Caucasian, G2P1001, presented at 40 weeks and 2 days gestation with regular contractions occurring every $7 \mathrm{~min}$. Patient reported good fetal movement and vaginal spotting without any leakage of fluid. Prior obstetrical history was significant for one full-term pregnancy that resulted in a vacuum assisted vaginal delivery of a 3,470 $\mathrm{g}$ infant, which was performed secondary to poor maternal expulsive efforts. The prenatal course was uncomplicated and the patient did not have any prior surgeries or significant past medical history.

On presentation to labor and delivery, the patient was noted to be $5 \mathrm{~cm}$ dilated, $50 \%$ effaced and -2 station. The patient was started on antibiotics for group B Streptococcus chemoprophylaxis. Five hours after presentation, amniorrhexis was performed when cervical exam revealed $8 \mathrm{~cm}$ dilation, $100 \%$ effacement and 0 station, with light meconium noted. Within the hour of amniorrhexis, the patient was determined to be fully dilated, with the head at 0 station. With an adequate epidural, the patient was expectantly managed for the next $2.5 \mathrm{~h}$. Fetal descent to +1 station was noted, but patient was without the urge to push. The fetal heart tracing remained category I during the early phase of her second stage of labor. Over the next $3 \mathrm{~h}$, there was an inadequate fetal descent, and with a short trial of pushing, the head descended to +2 station. The total duration of the second stage of labor was thus far $7 \mathrm{~h}$. After there was discussion with the patient regarding the protracted labor progress, oxytocin was started for augmentation. Shortly thereafter, fetal heart tracing displayed minimal variability and intermittent late decelerations. The patient suddenly complained of a new onset severe abdominal pain, despite a previously effective epidural anesthesia and the fetal tracing was notable for a prolonged fetal heart deceleration without any recovery in spite of intrauterine resuscitative efforts. The patient was immediately transferred to the operating room where an emergent primary low transverse cesarean section was performed. A female infant was delivered from an intraperitoneal, cephalic presentation with identification of a lower uterine segment rupture that had extended into the upper vagina. The female infant weighed 3,215 g and Apgars were 1,2 and 4 at 1, 5 and 10 min, respectively. Cord gases revealed umbilical artery $\mathrm{pH}$ of 6.84 with a base excess of -14.9 and umbilical vein $\mathrm{pH} 6.93$ with base excess of -13.3 . The uterus was repaired in a single layer with 0-monocryl suture (Fig. 1). The patient had an unremarkable postoperative course and was discharged home

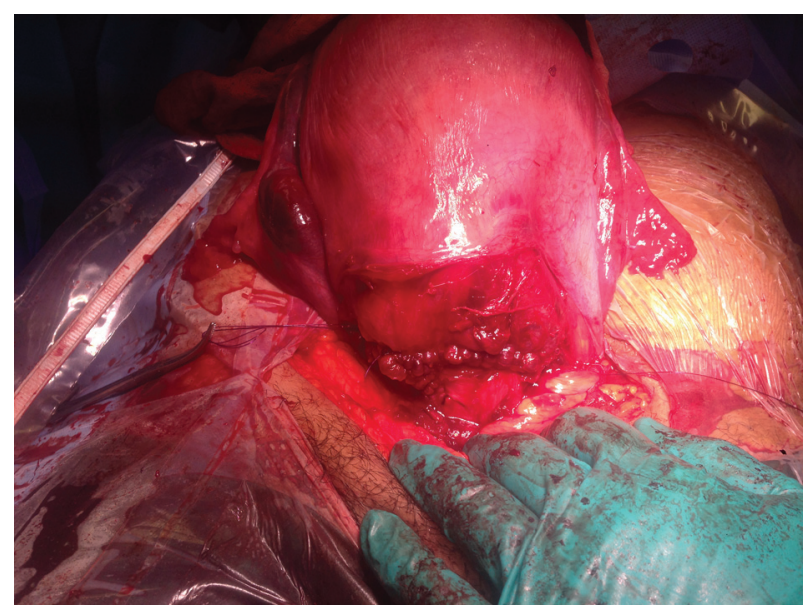

Figure 1. Uterus with repaired rupture site.

on postoperative day 4 .

\section{Discussion}

Rupture of the gravid uterus is a rare event, occurring in approximately 0.8 per 10,000 deliveries [1], causing significant maternal/fetal morbidity and mortality. Risk factors are poorly understood and may be divided into intrinsic and iatrogenic factors. Intrinsic factors include multiparity [2], advanced maternal age [3] and connective tissue disorders such as EhlersDanlos syndrome [4]. Among iatrogenic factors, previous uterine scar is the leading risk factor [5]. Other iatrogenic factors include prolonged labor [6] and the use of oxytocin which in one series was associated with a doubling of the risk of uterine rupture [5].

It is worth mentioning that the distinction between a "scarred" and "unscarred" uterus is poorly defined in the literature. There are reports which define "scarred" uterus as one in which any form of hysterotomy has previously occurred. This definition includes not only all types of caesarean uterine incisions but also other procedures in which the structural integrity of the myometrium has been disturbed. Prior myomectomy [7] has been identified as an etiological factor. Previous hysteroscopy or uterine curettage should not be regarded as uterine scarring [1] unless a recognized uterine perforation occurred. It should be noted that in one small series, two of the four patients with unscarred uterine rupture reported a history of uterine curettage [8]. In a separate study by Kaali et al, laparoscopy which was performed during the same session with curettage revealed a 10 times increase in the diagnosis of uterine perforation that would otherwise be missed [9]. Therefore, the clinician should be vigilant about the possibility of occult perforations for patients with prior hysteroscopy or curettage.

Diagnosis of uterine rupture remains very challenging as many of the clinical signs and symptoms lack a high degree of sensitivity or specificity. Abdominal pain may be difficult to be elicited in the presence of regional anesthesia and difficult to distinguish from labor pain [8]. Vaginal bleeding is often minimal and difficult to distinguish from other etiologies. The sen- 
sitivity of other signs of uterine rupture such as loss of station and/or contractions and uterine tenderness is also poor. Frequently, the constellation of signs and symptoms is difficult to distinguish those of uterine rupture from the more commonly encountered differential diagnoses such as chorioamnionitis, placental abruption or normal labor.

Vernixuria [10] has been described as a unique sign of uterine rupture. Maternal signs of hypotension and tachycardia frequently occur late in the process and lack sensitivity. Abnormal fetal heart rate tracing is inconsistent although many case series report fetal bradycardia as a frequently encountered event. The presence of an intra-uterine pressure catheter and subsequent alterations in readings has been suggested as a method of diagnosis for uterine rupture. However, this also lacks sensitivity [11].

The most important element for management of uterine rupture remains early recognition and high level of suspicion for diagnosis. Uterine rupture is a true obstetric emergency that requires prompt and immediate action. A multi-disciplinary team consisting of experienced obstetric surgeon, anesthesiology, operating room staff, neonatology and hematology should be assembled in a rapid manner. As significant hemorrhage is frequently encountered, a low threshold for activation of the massive transfusion protocol should be maintained. The principal surgical management involves early evacuation of the remaining uterine contents and delivery of both fetus and placenta. Next, the uterus should be completely evaluated to identify any defect that may be present. Surrounding structures such as the bladder, broad ligament and nearby bowel should also be inspected for possible injury. If non-gynecological structures are involved, then surgeons familiar with such structures should be consulted. The decision between repair of the uterine defect or hysterectomy should be dependent upon the degree of hemorrhage, the skill of the surgeon, the availability of equipment and the desire for future fertility.

The management of future pregnancies should be carefully considered after uterine rupture. The site of the rupture may influence the timing of a future delivery in that a fundal or corneal rupture may merit a delivery closer to 34 weeks whereas a lower segment rupture may merit delivery closer to 36 or 37 weeks. Amniocentesis to determine lung maturity should be considered.

\section{Conclusions}

Rupture of the unscarred uterus is an extremely rare and cata- strophic event. The risk factor profile is broad and the signs and symptoms lack sensitivity. As a consequence, we recommend that clinicians maintain a high index of suspicion for uterine rupture in the absence of prior uterine surgery. Further, when patients report a history of prior hysteroscopy or uterine curettage, clinicians should remain mindful of occult perforations that increase the risk of uterine rupture in the gravid uterus.

\section{References}

1. Zwart JJ, Richters JM, Ory F, de Vries JI, Bloemenkamp $\mathrm{KW}$, van Roosmalen J. Uterine rupture in The Netherlands: a nationwide population-based cohort study. BJOG. 2009;116(8):1069-1078; discussion 1078-1080.

2. Garnet JD. Uterine Rupture during Pregnancy. An Analysis of 133 Patients. Obstet Gynecol. 1964;23:898-905.

3. Hamilton BE, Martin JA, Sutton PD. National Vital Statistics Reports: Births: Preliminary data for 2003. Available at http://www.cdc.gov.nchs/data/nvsr/nvsr53/nvsr53_09. pdf.

4. Erez Y, Ezra Y, Rojansky N. Ehlers-Danlos type IV in pregnancy. A case report and a literature review. Fetal Diagn Ther. 2008;23(1):7-9.

5. Kaczmarczyk M, Sparen P, Terry P, Cnattingius S. Risk factors for uterine rupture and neonatal consequences of uterine rupture: a population-based study of successive pregnancies in Sweden. BJOG. 2007;114(10):1208-1214.

6. Sakr R, Berkane N, Barranger E, Dubernard G, Darai E, Uzan S. Unscarred uterine rupture--case report and literature review. Clin Exp Obstet Gynecol. 2007;34(3):190192.

7. Ahtorp G. On Conservative Myomectomy. Acta Obstet Gynecol Scand. 1946:26(Suppl):64-78.

8. Dow M, Wax JR, Pinette MG, Blackstone J, Cartin A. Third-trimester uterine rupture without previous cesarean: a case series and review of the literature. Am J Perinatol. 2009;26(10):739-744.

9. Kaali SG, Szigetvari IA, Bartfai GS. The frequency and management of uterine perforations during first-trimester abortions. Am J Obstet Gynecol. 1989;161(2):406-408.

10. O'Grady JP, Prefontaine M, Hoffman DE. Vernixuria: another sign of uterine rupture. J Perinatol. 2003;23(4):351352.

11. Rodriguez MH, Masaki DI, Phelan JP, Diaz FG. Uterine rupture: are intrauterine pressure catheters useful in the diagnosis? Am J Obstet Gynecol. 1989;161(3):666-669. 